\title{
Finite Element Analysis of Deformation and Stress Situation of Triangular Truss Under Different Loads
}

\author{
Zeng, WL (Zeng, Weiliang) $)^{1}$; Cong, Q (Cong, Qing) ${ }^{2}$, Liu, Y (Liu, Ying) $)^{[2,3, *]}$ \\ ${ }^{1}$ School of Mathematics Science, Harbin Normal University, Harbin, China \\ ${ }^{2}$ The Computing Center of Heilongjiang Province, Harbin, China \\ ${ }^{3}$ Information Center of Human Resources and Social Security of Heilongjiang Province , Harbin, China
}

\begin{abstract}
In this paper, the two methods for solving finite element problem, theoretical calculation method and ANSYS simulation analysis method, were used to study deformation and stress situation of each node of triangular truss under different distribution loads. The results of theoretical calculation were compared with those of ANSYS simulation, which showed that the counter-acting forces of each node were exactly the same. For the calculation results of the equivalent stiffness constants of each unit could not be accurate, values of each node deformation calculated by theoretical calculation could be error, but the maximum error rate was no more than $3.6 \%$. By the comparison results, conclusion could be made that the results of ANSYS simulation are more intuitive and image, the values are more accurate and reliable, comparing with those of theoretical calculation, the influence of accuracy of equivalent stiffness constant is much more smaller.
\end{abstract}

\section{Introduction}

Finite Element Method (FEM), is a kind of numerical calculation method for solving all kinds of engineering problems, which appeared in the late 1950s. In 1967, Zienkiewicz and Cheung published the first monograph on FEM (Zienkiewicz, 1967). FEM is an effective analysis method for solving problems that could not be solved by analytic method in the past, also it can solve complicated problems whose boundary conditions and structure shape are both irregular (Esen, 2013, Tang, 2013).

Truss is an engineering structure, composed of straight rods, connected together at the end by bolts, rivets, pins or welding. The straight rod of truss can be steel pipe, aluminum pipe, wood rod, metal bar, angle steel and channel steel. Truss is used to build traffic signal light, street lamp, transmission tower, bridge and roof of building. Members of the truss are usually regarded as two force rods, forces are equal in magnitude value and opposite in direction (Saeed, 2015).

This paper focused on research of triangular truss under different distribution loads. First, theoretical calculation of FEM was made, values of force and deformation of each node were got. Then, ANSYS simulation was made by ANSYS15.0, the results were compared with those obtained by theoretical calculation. Finally, reason of different of values was given.

\section{Calculation}

FEM is a numerical method which is widely used in solving engineering problems such as stress analysis, heat transfer, electromagnetic and fluid mechanics. The governing differential equations of practical engineering problems are quite complex, plus the boundary conditions and initial conditions, so obtaining analytic solutions is very difficult. By using FEM, the complexity of the structure is discretized into finite elements, which is based on the discretization of the problem domain into the elements and nodes, the selection of shape function, the establishment of element stiffness matrix, the combination of element stiffness matrix, the application of load, other boundary conditions and other steps, finally results are got by solving simultaneous linear equations (Pan Zeng, 2004).

The structure and size of triangular truss was shown in Fig. 1, all members were Q235 steel pipes, the diameter of steel tube $D$ was $40 \mathrm{~mm}$, the cross-sectional area $A$ was $1256.64 \mathrm{~mm}^{2}$, under normal temperature, the elastic modulus of Q235 steel pipe $E$ was $206000 \mathrm{MPa}$, Poisson ratio $v$ was 0.26 (Li Yongjun, 2011). There were two loads on the triangular truss in two different cases, the first one was $F 1=15000 \mathrm{~N}, F 2=15000 \mathrm{~N}$, the other one was $F 1=10000 N, \quad F 2=20000 N$. In each case, the deformation and stress situation of each node of triangular truss were calculated and the values were got. 


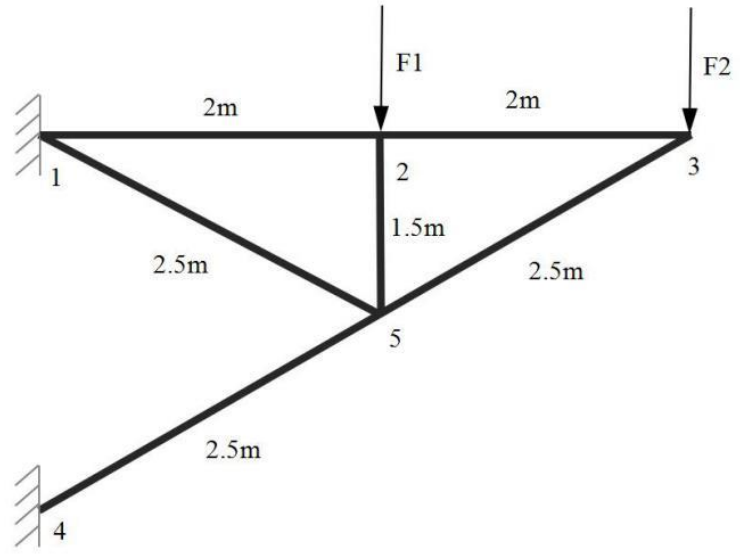

Figure 1. Schematic diagram of the force of triangle truss

The triangular truss was composed of five nodes, two nodes formed one unit, so there were six units, as Figure 1 showed. The lengths and angles of each unit were showed in Table 1.

Table 1. Relationship between the unit and the corresponding node

\begin{tabular}{|c|c|c|c|c|}
\hline Unit & Node-i & Node-j & $\begin{array}{c}\text { Length-L } \\
(\mathrm{m})\end{array}$ & Angle- $\alpha$ \\
\hline$(1)$ & 1 & 2 & 2 & $\sin \alpha=0, \cos \alpha=1$ \\
\hline$(2)$ & 2 & 3 & 2 & $\sin \alpha=0, \cos \alpha=1$ \\
\hline$(3)$ & 1 & 5 & 2.5 & $\begin{array}{c}\sin \alpha=-0.6 \\
\cos \alpha=0.8\end{array}$ \\
\hline$(4)$ & 2 & 5 & 1.5 & $\sin \alpha=-1, \cos \alpha=0$ \\
\hline$(5)$ & 3 & 5 & 2.5 & $\begin{array}{c}\sin \alpha=-0.6, \\
\cos \alpha=-0.8\end{array}$ \\
\hline$(6)$ & 4 & 5 & 2.5 & $\begin{array}{c}\sin \alpha=0.6, \\
\cos \alpha=0.8\end{array}$ \\
\hline
\end{tabular}

For Unit (1) and Unit (2), the equivalent stiffness constant $k_{l}$ was calculated as following:

$$
\begin{aligned}
& k_{1}=\frac{A E}{L}=\frac{1256.64 \mathrm{~mm}^{2} \times 2.06 \times 10^{5} \mathrm{MPa}}{2000 \mathrm{~mm}} \\
& \approx 1.3 \times 10^{5} \mathrm{~N} / \mathrm{mm}
\end{aligned}
$$

Similarly, according to Eq. 1, for Unit (3), Unit (5) and Unit (6), the approximate value $k_{2}$ was $1.0 \times 10^{5} \mathrm{~N} / \mathrm{mm}$, for Unit (4), the approximate value $k_{3}$ was $1.7 \times 10^{5} \mathrm{~N} / \mathrm{mm}$.

The element stiffness equation was built as following:

$$
K_{4 \times 4}^{(e)}=k\left[\begin{array}{cccc}
\cos ^{2} \alpha & \sin \alpha \cos \alpha & -\cos ^{2} \alpha & -\sin \alpha \cos \alpha \\
\sin \alpha \cos \alpha & \sin ^{2} \alpha & -\sin \alpha \cos \alpha & -\sin ^{2} \alpha \\
-\cos ^{2} \alpha & -\sin \alpha \cos \alpha & \cos ^{2} \alpha & \sin \alpha \cos \alpha \\
-\sin \alpha \cos \alpha & -\sin ^{2} \alpha & \sin \alpha \cos \alpha & \sin ^{2} \alpha
\end{array}\right]
$$

For Unit (1) and Unit (2), $\sin \alpha=0, \cos \alpha=1$, the local stiffness matrix $K^{(1,2)}$ was calculated by Eq. 2, as following:

$$
K^{(1,2)}=10^{5} \times\left[\begin{array}{cccc}
1.3 & 0 & -1.3 & 0 \\
0 & 0 & 0 & 0 \\
-1.3 & 0 & 1.3 & 0 \\
0 & 0 & 0 & 0
\end{array}\right]
$$

Similarly, for Unit (3), $\sin \alpha=-0.6, \cos \alpha=0.8$, the local stiffness matrix $K^{(3)}$ was calculated by Eq. 2, as following:

$$
K^{(3)}=10^{5} \times\left[\begin{array}{cccc}
0.64 & -0.48 & -0.64 & 0.48 \\
-0.48 & 0.36 & 0.48 & -0.36 \\
-0.64 & 0.48 & 0.64 & -0.48 \\
0.48 & -0.36 & -0.48 & 0.36
\end{array}\right]
$$

For Unit (4), $\sin \alpha=-1, \cos \alpha=0$, the local stiffness matrix $K^{(4)}$ was calculated by Eq. 2, as following:

$$
K^{(4)}=10^{5} \times\left[\begin{array}{cccc}
0 & 0 & 0 & 0 \\
0 & 1.7 & 0 & -1.7 \\
0 & 0 & 0 & 0 \\
0 & -1.7 & 0 & 1.7
\end{array}\right]
$$

For Unit (5), $\sin \alpha=-0.6, \cos \alpha=-0.8$, the local stiffness matrix $K^{(5)}$ was calculated by Eq. 2, as following:

$$
K^{(5)}=10^{5} \times\left[\begin{array}{cccc}
0.64 & 0.48 & -0.64 & -0.48 \\
0.48 & 0.36 & -0.48 & -0.36 \\
-0.64 & -0.48 & 0.64 & 0.48 \\
-0.48 & -0.36 & 0.48 & 0.36
\end{array}\right]
$$

For Unit (6), $\sin \alpha=0.6, \cos \alpha=0.8$, the local stiffness matrix $K^{(6)}$ was calculated by Eq. 2, as following:

$$
K^{(6)}=10^{5} \times\left[\begin{array}{cccc}
0.64 & 0.48 & -0.64 & -0.48 \\
0.48 & 0.36 & -0.48 & -0.36 \\
-0.64 & -0.48 & 0.64 & 0.48 \\
-0.48 & -0.36 & 0.48 & 0.36
\end{array}\right]
$$

All the local stiffness matrixes were added up together, then the global stiffness matrix $K^{(Q)}$ was got, as following:

$K^{(Q)}=10^{5} \times\left[\begin{array}{cccccccccc}1.94 & -0.48 & -1.3 & 0 & 0 & 0 & 0 & 0 & -0.64 & 0.48 \\ -0.48 & 0.36 & 0 & 0 & 0 & 0 & 0 & 0 & 0.48 & -0.36 \\ -1.3 & 0 & 2.6 & 0 & -1.3 & 0 & 0 & 0 & 0 & 0 \\ 0 & 0 & 0 & 1.7 & 0 & 0 & 0 & 0 & 0 & -1.7 \\ 0 & 0 & -1.3 & 0 & 1.94 & 0.48 & 0 & 0 & -0.64 & -0.48 \\ 0 & 0 & 0 & 0 & 0.48 & 0.36 & 0 & 0 & -0.48 & -0.36 \\ 0 & 0 & 0 & 0 & 0 & 0 & 0.64 & 0.48 & -0.64 & -0.48 \\ 0 & 0 & 0 & 0 & 0 & 0 & 0.48 & 0.36 & -0.48 & -0.36 \\ -0.64 & 0.48 & 0 & 0 & -0.64 & -0.48 & -0.64 & -0.48 & 1.92 & 0.48 \\ 0.48 & -0.36 & 0 & -1.7 & -0.48 & -0.36 & -0.48 & -0.36 & 0.48 & 2.78\end{array}\right]$

When the global stiffness matrix $K^{(Q)}$ and applied force $F$ were got, the following formula equation Eq. 3 could be used to solve equation and get the nodal displacement $U$.

$$
K^{(Q)} U=F
$$

As Figure 1 shown, because Node (1) and Node (4) were fixed, $U_{1 X}=0, U_{1 Y}=0, U_{4 X}=0, U_{4 Y}=0$, there were only down loads on node 2 and node 3 .

When external load $F 1=15000 N, F 2=15000 N$, then $F_{2 Y}=-15000 N, \quad F_{3 Y}=-15000 N$, the linear system of equations was got by Eq. 3 as following:

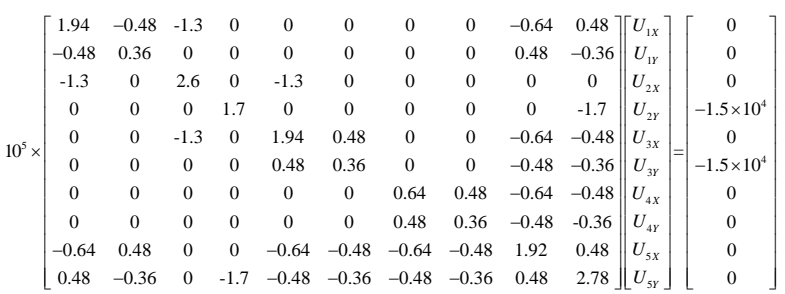


For $U_{I X}=0, U_{I Y}=0, U_{4 X}=0, U_{4 Y}=0$, the line No.1, No.2, No.7, No.8 and the column No.1, No.2, No.7, No.8 are deleted, the matrix equation was got as following:

$10^{5} \times\left[\begin{array}{cccccc}2.6 & 0 & -1.3 & 0 & 0 & 0 \\ 0 & 1.7 & 0 & 0 & 0 & -1.7 \\ -1.3 & 0 & 1.94 & 0.48 & -0.64 & -0.48 \\ 0 & 0 & 0.48 & 0.36 & -0.48 & -0.36 \\ 0 & 0 & -0.64 & -0.48 & 1.92 & 0.48 \\ 0 & -1.7 & -0.48 & -0.36 & 0.48 & 2.78\end{array}\right]\left[\begin{array}{c}U_{2 X} \\ U_{2 Y} \\ U_{3 X} \\ U_{3 Y} \\ U_{5 X} \\ U_{5 Y}\end{array}\right]=\left[\begin{array}{c}0 \\ -1.5 \times 10^{4} \\ 0 \\ -1.5 \times 10^{4} \\ 0 \\ 0\end{array}\right]$

The results were got after solving system of linear equations, as following: $U_{2 X}=0.1538 \mathrm{~mm}, \quad U_{2 Y}=-$ $0.5049 \mathrm{~mm}, \quad U_{3 X}=0.3077 \mathrm{~mm}, \quad U_{3 Y}=-1.4519 \mathrm{~mm}, \quad U_{5 X}=-$ $0.1563 \mathrm{~mm}, U_{5 Y}=-0.4167 \mathrm{~mm}$.

Because that Node (1) and Node (4) were fixed, $U_{I X}=0, U_{1 Y}=0, U_{4 X}=0, U_{4 Y}=0$, so the result of $U$ were got. The counter-acting forces could be calculated by Eq. 4, as following:

$$
P=K^{(Q)} U-F
$$

Using parameters $K^{(Q)}, U, F$ in Eq. 4 , the counteracting force $P$ was got as following: $P_{I X}=-29992 N$, $P_{1 Y}=7499 \mathrm{~N}, \quad P_{4 X}=30005 \mathrm{~N}, \quad P_{4 Y}=22504 \mathrm{~N}$. For the calculation results of the equivalent stiffness constants of each unit could not be accurate, resulting in error, so other values were zero.

Also, when external load $F 1=10000 \mathrm{~N}, \mathrm{~F} 2=20000 \mathrm{~N}$, then $F 2 Y=-10000 N, F 3 Y=-20000 N$, the results of the nodal displacement $\mathrm{U}$ were got after solving the system of linear equations, as following:

$\mathrm{U} 2 \mathrm{X}=0.2051 \mathrm{~mm}$,

$$
\mathrm{U} 2 \mathrm{Y}=-0.4755 \mathrm{~mm} \text {, }
$$
$\mathrm{U} 3 \mathrm{X}=0.4103 \mathrm{~mm}, \mathrm{U} 3 \mathrm{Y}=-1.7970 \mathrm{~mm}, U_{5 X}=-0.2083 \mathrm{~mm}$, $U_{5 Y}=-0.4167 \mathrm{~mm}$.

Similarly, the values of counter-acting force $P$ were got as following: $P_{I X}=-33333 N, \quad P_{I Y}=5003 N$, $P_{4 X}=33333 N, P_{4 Y}=25000 N$.

\section{ANSYS Simulation}

ANSYS is a large general finite element analysis (FEA) software developed by ANSYS company of U.S.A and is the fastest growing computer aided engineering (CAE) software in the world. ANSYS is powerful and easy to operate, it has now become one of the most popular finite element analysis software in the world.

Using ANSYS15.0 to carry on the analysis of the force and deformation of triangle truss under different loads, simulation diagram of force distribution on triangle truss was shown in Figure 2.

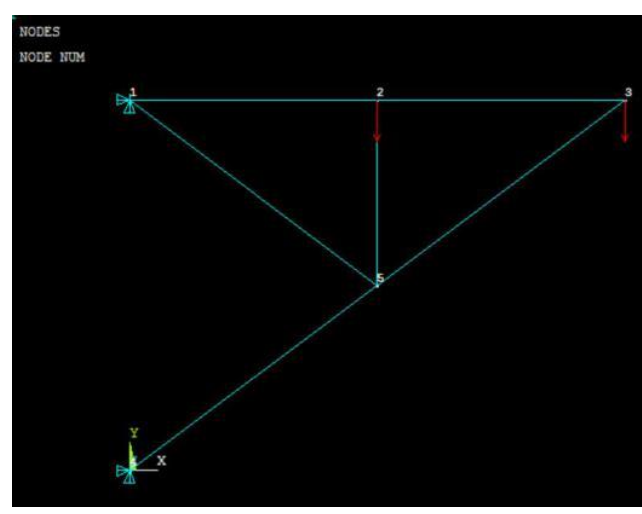

Figure 2. ANSYS simulation diagram of force distribution

When external load $F 1=15000 N, F 2=15000 N$, then $F_{2 Y}=-15000 \mathrm{~N}, F_{3 Y}=-15000 \mathrm{~N}$, from ANSYS simulation, after pro-process, solution and post-process, values of $U_{X}$ and $U_{Y}$ of each node were got, values of nodal displacement were got, diagram of node deformation was shown in Figure 3.

Deformation of Nodes were got: $U_{2 X}=0.1545 \mathrm{~mm}$, $U_{2 Y}=-0.4893 \mathrm{~mm}, \quad U_{3 X}=0.3090 \mathrm{~mm}, \quad U_{3 Y}=-1.4180 \mathrm{~mm}$, $U_{5 X}=-0.1509 \mathrm{~mm}, U_{5 Y}=-0.4024 \mathrm{~mm}$. The counter-acting forces could be obtained as: $P_{I X}=-30000 N, P_{I Y}=7500 N$, $P_{4 X}=30000 \mathrm{~N}, P_{4 Y}=22500 \mathrm{~N}$, they were in full agreement with those of theoretical calculation .

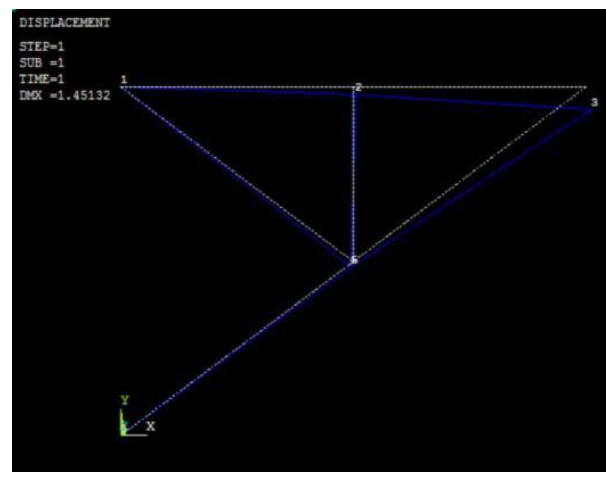

Figure 3. ANSYS simulation diagram of node deformation of triangle truss

When external load $F 1=10000 \mathrm{~N}, F 2=20000 \mathrm{~N}$, then $F_{2 Y}=-10000 \mathrm{~N}, F_{3 Y}=-20000 \mathrm{~N}$, after ANSYS simulation, diagram of node deformation was shown in Figure 4.

Deformation of Nodes were got: $U_{2 x}=0.20603 \mathrm{~mm}$, $U_{2 Y}=-0.46034 \mathrm{~mm}, \quad U_{3 X}=0.412 \mathrm{~mm}, \quad U_{3 Y}=-1.7566 \mathrm{~mm}$, $U_{5 X}=-0.2012 \mathrm{~mm}, U_{5 Y}=-0.40239 \mathrm{~mm}$. The counter-acting forces could be obtained as: $P_{I X}=-33333 N, P_{I Y}=5000 N$, $P_{4 X}=33333 N, P_{4 Y}=25000 N$, they were in full agreement with those of theoretical calculation . 


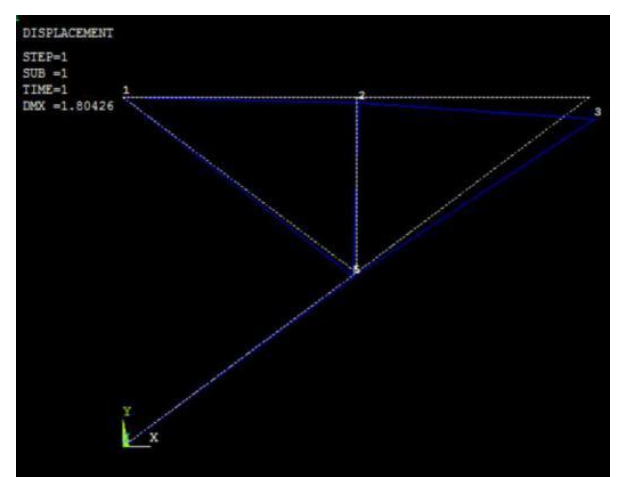

Figure 4. ANSYS simulation diagram of node deformation of triangle truss

\section{Conclusions}

In the paper, the two methods, theoretical calculation method of finite element and ANSYS simulation analysis method, were used to study the deformation and stress situation of each node of triangular truss under different distribution loads. The results of theoretical calculation were compared with those of ANSYS simulation, that showed that the counter-acting force of each node counter force were exactly the same. For the calculation results of the equivalent stiffness constants of each unit could not be accurate, nodal displacement calculated by theoretical calculation could be error, but the maximum error rate was no more than $3.6 \%$. By the comparison results, conclusion could be made that the results of ANSYS simulation were more intuitive and image, its values were more accurate and reliable, compared with those of theoretical calculation, because that the influence of accuracy of equivalent stiffness constant was much more smaller.

\section{Acknowledgements}

This research was financially supported by Special Fund Project for Scientific Innovation Personnel Research of Harbin (2013RFQXJ088), Foundation for University Key Teacher of Heilongjiang Province of China (1253G035) and the Pre-research Funds of Harbin Normal University (XKGP21201305).

\section{References}

1. Zienkiewicz, O. C., and Cheung, Y. K. K., The Finite Element Method in Structural and Continuum Mechanics, London, McGraw-Hill, 1967.

2. Esen. A, Ucar. Y, Yagmurlu. N, Tasbozan. O, A Galerkin Finite Element Method to Solve Fractional Diffusion and Fractional Diffusion-Wave Equations, Mathematical Modelling and Analysis, 18(2), (2013), 260-273.

3. Tang, YL, An Improved Finite Element Approximation and Superconvergence for Temperature Control Problems, Mathematical Modelling and Analysis, 18(5), 2013, 631-640.
4. Saeed Moaveni, Finite Element Analysis: Theory and Application with ANSYS (Fourth Edition), PEARSON EDUCATION, 2015.

5. Pan Zeng, Fundamentals of Finite Element Analysis, Tsinghua University Press, 2004.

6. Li Yongjun, Ma Liyuan, Li Shilong, Wang Tianhui. Modeling and Model Amendment for Welded Steel Tube with Parameters Object, Chinese Journal of Mechanical Engineering, 22(22), (2011), 2754-2757. 